\title{
Sentinel lymph node in early cervical cancer - pros and cons aspects
}

\author{
Alice SAVESCU ${ }^{1}$, Irina BALESCU ${ }^{2}$, Nicolae BACALBASA ${ }^{3}$ \\ ${ }^{1}$ Department of Obstetrics and Gynecology, University Emergency Hospital, Bucharest, Romania \\ ${ }_{2}^{2}$,Ponderas“ Hospital, Bucharest, Romania \\ 3 "Carol Davila“ University of Medicine and Pharmacy, Bucharest, Romania
}

\begin{abstract}
Cervical cancer remains an important health problem worldwide responsible for a high number of deaths especially in cases diagnosed in advanced stages. When it comes to patients diagnosed in early stages, they have an improved prognosis with high rates of 5 year overall survival. Due to the fact that one of the most important routes of dissemination remains the lymphatic pattern, studies were focused on determining which cases are more suitable for lymph node dissection even if an early stage of the disease is expected. This is a literature review regarding pros and cons aspects of the utility of sentinel lymph node in early stage cervical cancer.
\end{abstract}

Keywords: early stage, cervical cancer, sentinel lymph node, lymph node dissection

\section{INTRODUCTION}

The incidence of cervical cancer in central and eastern Europe is around 14.5\%, being the third most commonly diagnosed cancer in women with a mortality rate of $6.3 \%$, which makes it the fourth leading cause of cancer death in females worldwide (1). When diagnosed in an early stage such as FIGO Stage 0-IB, the 5-year survival rate ranks between 80 and $93 \%$, while in advanced stages the 5 -year survival rate is lower than $40 \%$ (2); therefore a proper staging and adequate treatment is mandatory.

The role of pelvic lymph node dissection in early stage cervical cancer

The standard treatment of early cervical cancer is radical abdominal hysterectomy and pel- vic lymphadenectomy (3). The evaluation of lymph node involvement remains an important procedure due to the fact that lymph node status is one of the most important prognostic factors in patients diagnosed with early stage cervical cancer. When lymph node involvement is present the 5-year survival rate becomes lower (65.1\%) compared with 5-year survival rate in the patients without metastatic lymph node (93.2\%) (4). Although pelvic lymphadenectomy associated with radical hysterectomy remains the gold standard treatment, pelvic node metastasis are identified in approximately one third of the cases, varying between $12 \%-26 \%$ (57). However, lymph node dissection is associated with complications including symptomatic postoperative lymphocysts, lower-limb lymphedema, nerve injury (obturator neuropathy) and urological dysfunction (8-10). 
The concept of sentinel lymph node in cervical cancer

The sentinel lymph node is the first lymph node that receives drainage from the primary tumor, so it is the most likely area where cancer cells might be found (11). The use of sentinel lymph node represents the standard of care for the management of several malignancies, such as melanoma and breast cancer, while the role of sentinel lymph node in cervical cancer has not been established yet (12). However, once the concept of sentinel lymph node has been introduced, an unnecessary lymph node dissection has been avoided and consequently the incidence of postoperative morbidity decreased.

\section{Techniques of lymphatic mapping}

Several lymphatic mapping techniques have been described: the blue dye is the first described technique. There are several different blue dyes that are commonly used (isosulfan blue, methylene blue, patent blue). The substance is injected under general anesthesia into each quadrant of the cervix (at 12, 3, 6 and 9 o'clock) or only at 3 and 9 o'clock. The sentinel lymph node becomes bluish and can be observed by naked eye. Although the negative predictive values mentioned were good varying from $88 \%-100 \%$, the sensitivity was inconsistent ranging from $94 \%-100 \%$ (13-15). When patent blue is used the mapping technique is associated with high false-negative rate because it has a short wash out time and may be drained in the second lymph node, the so called "false sentinel node" (15).

Patients may undergo sentinel lymph node biopsy using radioisotope technetium Tc $99 \mathrm{~m}$ sulfur colloid tracer detected with a hand-held gamma probe intraoperative. According to the timing of radio-colloid injection the tracer is injected intracervically the day prior to surgery, in the morning with only a few hours prior to surgery or intraoperatively (16-18). Detection of sentinel lymph node is significantly better when blue dye technique is associated with Tc $99 \mathrm{~m}$ mapping (18).

A novel concept is the use of fluorescent dyes such as indocyanine green in association with near infrared visualization cameras. Studies mentioned a detection rate ranking between $83 \%-88 \%(19,20)$. Because detection rate is similar to that obtained with the blue dye and radioisotope method, indocyanine green technique is considered to be effective for sentinel lymph node detection (21). However, further studies, including combined techniques with blue dye are required to establish this mapping procedure as a reliable one.

The most common location of sentinel lymph node was interiliac in $71 \%$ (22), external iliac and interiliac areas in $80.6 \%$, but also aberrant drainage patterns were identified in $17 \%$ (23). In an attempt to identify the sentinel lymph nodes more accurately, preoperative diagnostic methods have been described. Preoperative sentinel node mapping with planar lymphoscintigraphy uses blue dye and technetium Tc $99 \mathrm{~m}$ on the basis of "long" protocol followed by intraoperative lymphatic mapping with gamma probe. It has a poor detection regarding laterality, number and location of sentinel lymph nodes (24), so it has scarce clinical value (25). Single photon emission computed tomography (SPECT) is another nuclear medicine imaging technique that uses gamma rays providing three-dimensional imaging. SPECT is usually associated with computed tomography (CT) scan and seems to offer an improvement in both detection and anatomic localization of sentinel lymph node $(26,27)$; in the meantime the method reduces intraoperative time for sentinel node retrieval (28). Interstitial magnetic resonance (MR) lymphography uses albumin-Gd-DTA as a contrast agent to identify hot areas of the lymphatic drainage so called 'pooling' zones. This method has an accuracy in detecting lymph node of $92.9 \%$, with a sensitivity and specificity of $80 \%$ and $95.7 \%$ comparable with SPECT/CT, therefore should be routinely performed preoperatively (29).

Sentinel lymph node identification was successfully performed in early cervical cancer when tumors were smaller than $2 \mathrm{~cm}$ with a negative predictive value for these patients ranking between $94.2 \%$ and $100 \%$. In case of bilateral sentinel lymph node complete pelvic lymphadenectomy could be avoided; however in cases in which only unilateral sentinel lymph node is detected a complete lymphadenectomy should be performed on the other side where no radioactive or dye signal has been registered. $(30,31)$ An algorithm to detect positive sentinel lymph node for early cervical cancer (FIGO stages IA1 with lymph vascular space invasion to IIA) was developed by the Memorial Sloan Kettering Cancer Center (MSKCC); the technique seems to have a comprehensive detection of all patients with positive lymph nodes and complete pelvic lymphadenectomy can be avoided in the majority of cases. The surgical algorithm evaluated by MSKCC's team included the following: removal 
of sentinel lymph nodes subsequently submitted to ultrastaging, removal of any suspicious lymph node regardless of mapping, performing contralateral side-specific pelvic lymphatic node dissection (including inter-iliac nodes) if only unilateral lymph node is noted and in all cases performing parametrectomy en bloc with primary tumor resection. (32) Tests to establish the feasibility of the algorithm were taken and false negative results were noted in $10 \%$ of patients. (33) However, additional studies are still needed to validate the algorithm as a safe one.

Once sentinel lymph node is detected, it is typically sent to histopathological examination with frozen section or imprint cytology. The tissue is routinely submitted to hematoxylin and eosin staining. However, although large studies have investigated the accuracy of frozen sections in detecting positive lymph nodes, the results are not promising, this procedure being associated with low sensitivity and poor diagnostic value. (34)

One of the main problem was its inability of detecting micrometastases (to be $>0.2 \mathrm{~mm}$ but not $>2.0 \mathrm{~mm}$ ) and isolated tumor cells $(\leq 0.2$ $\mathrm{mm}$ ). For these reasons the ultrastaging concept was developed. This concept consists in serial sectioning (step-serial sectioning 3-microm thick slides at 200-500 microm intervals) followed by immunohistochemistry staining with an anti-cytokeratin antibody (AE1/AE3). By this method a low overall false-negative rate and an even lower false-negative rate were achieved when bilateral mapping was done. In the meantime the negative prognostic value was higher (100\%) after ultrastaging comparing with a $94.2 \%$ negative prognostic value provided by frozen section. (30) Other studies revealed even a lower negative prognostic value of $93 \%$ on intraoperative frozen section. (34) The false negative rate of frozen section was higher among patients with low-volume disease greater than $20 \mathrm{~cm}^{3}$. Ultastaging seems to be more reliable at detecting micrometastases than routine hematoxylin and eosin histology (35), but it can't be applied to intraoperative histological examination.

Therefore the need for a quick, reliable, and reproducible method for lymph node analysis appears (36). Beginning from these premises, one-step nucleic acid amplification (OSNA) assay that uses cytokeratin (CK) 19 (KRT19) messenger RNA (mRNA) was introduced. This method is already in use for breast carcinoma; however for early cervical cancer it is not vali- dated yet (36). The role of high-risk types of the human papillomaviruses (hr-HPV) such as HPV16 in the development of cervical cancer is well established, but his role in sentinel lymph node is questionable. Messenger RNA expression patterns of E6 gene transcripts were quantified in sentinel lymph node by a reverse transcriptase-PCR method (RT-PCR) along with another molecular marker cytokeratin (CK) 19. The authors concluded that HPV16 E6 mRNA is more specific and more sensitive for the detection of positive cells in sentinel lymph node than CK19 mRNA (37).

Micrometastases are identifiable in negative lymph nodes in $15 \%$ when ultrastaging technique is used. However their presence is not associated with recurrence, nor with a poor prognosis. $(38,39)$ An association between micrometastases and lympho-vascular space invasion was establish, therefore these group may benefit from adjuvant chemo-irradiation. (35) A survival improvement was noted when more than 16 additional non sentinel lymph node were dissected along with postitive of low-volume disease sentinel lymph node. (40) At this moment the role of micrometastases in early cervical cancer is under close observation, its' prognostic value being under surveillance.

\section{The role of sentinel lymph node in fertility sparing surgery}

The latest trend around young women is toward late childbearing and when they are faced with an early cervical cancer diagnosis fertilitypreserving procedure become a major issue. (41) Suitable candidates for fertility preservation are those with a certain pathologic type of disease and stage. For example FIGO Stage IA1 with depth of cervical stromal invasion less than $3 \mathrm{~mm}$ is effectively treated by cervical conisation (42), while FIGO Stage IA1 with lymph vascular space invasion, FIGO Stage IA2 and FIGO Stage IB1 cancers less than $2 \mathrm{~cm}$ are treated by radical trachelectomy (41). Radical trachelectomy can be performed either by abdominal or vaginal route, in association with laparoscopic pelvic lymphadenectomy. More recently the concept of minimally invasive surgery in cervical cancer has been extended because it was shown that only a small subgroup of patients ranking between $0.4 \%-0.6 \%$ report parametrial disease. (43-45) Low-risk characteristics for parametrial spread include women with negative lymph nodes, no lymph vascular space invasion, tumors smaller than $2 \mathrm{~cm}$ and stromal invasion 10 $\mathrm{mm}$ or less. 
Sentinel lymph concept is now associated with fertility-preserving surgical procedure. Rob et al described large cone or simple vaginal trachelectomy on 26 patients with FIGO Stage IA2 and Stage IB1 who desire to maintain fertility combined with laparoscopic pelvic lymphadenectomy and sentinel lymph node identification. All sentinel lymph nodes were submitted for ultrastaging. Only one recurrence was observed (46). In another study the sensitivity of sentinel lymph nodes using technetium Tc $99 \mathrm{~m}$ in detecting pelvic lymph nodes disease for radical abdominal trachelectomy was $100 \%$. Two recurrences occurred in 68 women with FIGO Stage IA2-IB1. (47) Another study that marks the usefulness of less invasive oncologic procedure (in selected women with small-volume FIGO Stage I) such as cervical conisation and sentinel lymph node mapping as a fertility-sparing technique. (48) Although some dates exist, further studies are required to establish the exact utility of less invasive surgery in early cervical.

\section{CONCLUSION}

Although the heterogeneity of the studies is relatively high it seems that sentinel lymph node concept in cervical cancer is feasible and useful in predicting nodal disease.

\section{REFERENCES}

1. Jemal A., Bray F., Center M.M. et al. Global cancer statistics. CA Cancer J Clin. 2011; 61:69-90

2. http://www.cancer.org/cancer/cervical cancer/detailedguide/cervical-cancersurvival, Survival rates for cervical cancer, by stage

3. Abu-Rustum N.R., Hoskins W.J. - Radical abdominal hysterectomy. Surg Clin North Am. 2001; 81:815-828

4. Zhang H.Y., Sheng X.G., Zhong Y. et al. (The rule of metastatic pelvic lymph node distribution in patients with early stage cervical carcinoma). Zhonghua Zhong Liu Za Zhi. 2008; 30:452-455

5. Benedetti-Panici P., Maneschi F., Scambia G. et al. - Lymphatic spread of cervical cancer: an anatomical and pathological study based on 225 radical hysterectomies with systematic pelvic and aortic lymphadenectomy. Gynecol Oncol. 1996; 62:19-24

6. Sakuragi N., Satoh C., Takeda N. et al. Incidence and distribution pattern of pelvic and paraaortic lymph node metastasis in patients with Stages IB, IIA, and IIB cervical carcinoma treated with radical hysterectomy. Cancer 1999; 85:1547-1554

7. Michel G., Morice P., Castaigne D. et al. Lymphatic spread in stage Ib and II cervical carcinoma: anatomy and surgical implications. Obstet Gynecol. 1998; 91:360-363

8. Achouri A., Huchon C., Bats A.S. et al. Complications of lymphadenectomy for gynecologic cancer. Eur J Surg Oncol. 2013; 39:81-86

9. Ricciardi E., Jakimovska M., Maniglio P. et al. - Laparoscopic injury of the obturator nerve during fertility-sparing procedure for cervical cancer. World J Surg Oncol. 2012; 10:177
10. Matsuura Y., Kawagoe T., Toki N. et al. Long-standing complications after treatment for cancer of the uterine cervix - clinical significance of medical examination at 5 years after treatment. Int J Gynecol Cancer. 2006; 16:294-297

11. Holman L.L., Levenback C.F., Frumovitz M. - Sentinel lymph node evaluation in women with cervical cancer. J Minim Invasive Gynecol. 2014; 21:540545

12. Altgassen C., Hertel H., Brandstadt A. et al. - Multicenter validation study of the sentinel lymph node concept in cervical cancer: AGO Study Group. J Clin Oncol. 2008; 26:2943-2951

13. Schwendinger V., Muller-Holzner E., Zeimet A.G. et al. - Sentinel node detection with the blue dye technique in early cervical cancer. Eur J Gynaecol Oncol. 2006; 27:359-362

14. O'Boyle J.D., Coleman R.L., Bernstein S.G. et al. - Intraoperative lymphatic mapping in cervix cancer patients undergoing radical hysterectomy: A pilot study. Gynecol Oncol. 2000; 79:238-243

15. Marchiole P., Buenerd A., Scoazec J.Y. et al. - Sentinel lymph node biopsy is not accurate in predicting lymph node status for patients with cervical carcinoma. Cancer. 2004; 100:2154-2159

16. Kato H., Todo Y., Minobe S. et al. Previous conization on patient eligibility of sentinel lymph node detection for early invasive cervical cancer. Int J Gynecol Cancer. 2011; 21:1491-1494

17. Gortzak-Uzan L., Jimenez W., NofechMozes S. et al. - Sentinel lymph node biopsy vs. pelvic lymphadenectomy in early stage cervical cancer: is it time to change the gold standard? Gynecol Oncol. 2010; 116:28-32
18. Rob L., Strnad P., Robova H. et al. - Study of lymphatic mapping and sentinel node identification in early stage cervical cancer. Gynecol Oncol. 2005; 98:281-288

19. Furukawa N., Oi H., Yoshida S. et al. The usefulness of photodynamic eye for sentinel lymph node identification in patients with cervical cancer. Tumori. 2010; 96:936-940

20. Rossi E.C., Ivanova A., Boggess J.F. Robotically assisted fluorescence-guided lymph node mapping with ICG for gynecologic malignancies: a feasibility study. Gynecol Oncol. 2012; 124:78-82

21. Xiong L., Gazyakan E., Yang W. et al. Indocyanine green fluorescence-guided sentinel node biopsy: a meta-analysis on detection rate and diagnostic performance. Eur J Surg Oncol. 2014; 40:843-849

22. Marnitz S., Kohler C., Bongardt S. et al. Topographic distribution of sentinel lymph nodes in patients with cervical cancer. Gynecol Oncol. 2006; 103:35-44

23. Bats A.S., Mathevet P., Buenerd A. et al. - The sentinel node technique detects unexpected drainage pathways and allows nodal ultrastaging in early cervical cancer: insights from the multicenter prospective SENTICOL study. Ann Surg Oncol. 2013; 20:413-422

24. Fotiou S., Zarganis P., Vorgias G. et al. - Clinical value of preoperative lymphoscintigraphy in patients with early cervical cancer considered for intraoperative lymphatic mapping. Anticancer Res. 2010; 30:183-188

25. Frumovitz M., Coleman R.L., Gayed I.W. et al. - Usefulness of preoperative lymphoscintigraphy in patients who undergo radical hysterectomy and pelvic lymphadenectomy for cervical cancer. Am J Obstet Gynecol. 2006; 194:1186-1193 
26. Pandit-Taskar N., Gemignani M.L., Lyall

A. et al. - Single photon emission computed tomography SPECT-CT improves sentinel node detection and localization in cervical and uterine malignancy. Gynecol Oncol. 2010; 117:59-64

27. Buda A., Elisei F., Arosio M. et al. Integration of hybrid single-photon emission computed tomography/computed tomography in the preoperative assessment of sentinel node in patients with cervical and endometrial cancer: our experience and literature review. Int J Gynecol Cancer. 2012; 22:830-835

28. Hoogendam J.P., Hobbelink M.G., Veldhuis W.B. et al. - Preoperative sentinel node mapping with $(99 \mathrm{~m})$ Tc-nanocolloid SPECT-CT significantly reduces the intraoperative sentinel node retrieval time in robot assisted laparoscopic cervical cancer surgery. Gynecol Oncol. 2013; 129:389-394

29. Hong Y., Xiang L., Hu Y. et al. - Interstitial magnetic resonance lymphography is an effective diagnostic tool for the detection of lymph node metastases in patients with cervical cancer. BMC Cancer. 2012;12:360.

30. Roy M., Bouchard-Fortier G., Popa I. et al. Value of sentinel node mapping in cancer of the cervix. Gynecol Oncol. 2011; 122:269274

31. Darlin L., Persson J., Bossmar T. et al. The sentinel node concept in early cervical cancer performs well in tumors smaller than 2 cm. Gynecol Oncol. 2010; 117:266-269

32. Cormier B., Diaz J.P., Shih K. et al. Establishing a sentinel lymph node mapping algorithm for the treatment of early cervical cancer. Gynecol Oncol. 2011; 122:275-280

33. de Freitas R.R., Baiocchi G., Hatschbach S.B. et al. - Can a sentinel node mapping algorithm detect all positive lymph nodes in cervical cancer? Ann Surg Oncol. 2015; 22:1564-1569

34. Bats A.S., Buenerd A., Querleu D. et al. Diagnostic value of intraoperative examination of sentinel lymph node in early cervical cancer: a prospective, multicenter study. Gynecol Oncol. 2011; 123:230-235

35. Juretzka M.M., Jensen K.C., Longacre T.A. et al. - Detection of pelvic lymph node micrometastasis in stage IA2-IB2 cervical cancer by immunohistochemical analysis. Gynecol Oncol. 2004; 93:107-111

36. Okamoto S., Niikura H., Nakabayashi K. et al. - Detection of sentinel lymph node metastases in cervical cancer: assessment of KRT19 mRNA in the one-step nucleic acid amplification (OSNA) method. Gynecol Oncol. 2013; 130:530-536

37. Hafner N., Gajda M., Altgassen C. et al. HPV16-E6 mRNA is superior to cytokeratin 19 mRNA as a molecular marker for the detection of disseminated tumour cells in sentinel lymph nodes of patients with cervical cancer by quantitative reversetranscription PCR. Int J Cancer. 2007; 120:1842-1846

38. Stany M.P., Stone P.J., Felix J.C. et al. - Lymph Node Micrometastases in Early-Stage Cervical Cancer are Not Predictive of Survival. Int J Gynecol Pathol. 2015; 34:379-384

39. Lentz S.E., Muderspach L.I., Felix J.C. et al. - Identification of micrometastases in histologically negative lymph nodes of early-stage cervical cancer patients. Obstet Gynecol. 2004; 103:1204-1210

40. Zaal A., Zweemer R.P., Zikan M. et al. Pelvic lymphadenectomy improves survival in patients with cervical cancer with low-volume disease in the sentinel node: a retrospective multicenter cohort study. Int J Gynecol Cancer. 2014; 24:303-311

41. Martinez A., Poilblanc M., Ferron G. et al. - Fertility-preserving surgical procedures, techniques. Best Pract Res Clin Obstet Gynaecol. 2012; 26:407-424

42. Kyrgiou M., Koliopoulos G., Martin-Hirsch P. et al. - Obstetric outcomes after conservative treatment for intraepithelial or early invasive cervical lesions: systematic review and metaanalysis. Lancet. 2006; 367:489-498

43. Wright J.D., Grigsby P.W., Brooks R. et al. - Utility of parametrectomy for early stage cervical cancer treated with radical hysterectomy. Cancer. 2007; 110:12811286

44. Frumovitz M., Sun C.C., Schmeler K.M. et al. - Parametrial involvement in radical hysterectomy specimens for women with early-stage cervical cancer. Obstet Gynecol. 2009; 114:93-99

45. Covens A., Rosen B., Murphy J. et al. How important is removal of the parametrium at surgery for carcinoma of the cervix? Gynecol Oncol. 2002; 84:145-149

46. Rob L., Charvat M., Robova H. et al. Less radical fertility-sparing surgery than radical trachelectomy in early cervical cancer. Int J Gynecol Cancer. 2007; 17:304-310

47. Du X.L., Sheng X.G., Jiang T. et al. Sentinel lymph node biopsy as guidance for radical trachelectomy in young patients with early stage cervical cancer. BMC Cancer. 2011; 11:157

48. Andikyan V., Khoury-Collado F., Denesopolis J. et al. - Cervical conization and sentinel lymph node mapping in the treatment of stage I cervical cancer: is less enough? Int J Gynecol Cancer. 2014; 24:113-117 